\title{
Unsteady Two-Dimensional MHD Flow of a Visco-elastic Fluid along Vertical Porous Surface with Heat Source and Chemical Reaction
}

\author{
T. Parida ${ }^{1}$, P. K. Rath ${ }^{2}$ \\ ${ }^{1}$ Dept. of Mathematics, College of Engineering Bhubaneswar, Bhubaneswar-751024, Odisha, India \\ ${ }^{2}$ Dept. of Mathematics, B.R.M. International Institute of Technology, Bhubaneswar-751010, Odisha, India
}

\begin{abstract}
An unsteady two dimensional magnetohydrodynamic flow of a visco-elastic incompressible fluid (Walters $B^{\prime}$ fluid model) along an infinite hot vertical porous surface bounded by porous medium with free stream as well as suction velocity in the presence of heat surface and chemical reaction has been investigated. The governing equations of motion, energy and concentration are solved by the successive perturbation technique. The flow phenomenon has been characterized by magnetic parameter $M$, elastic parameter $K_{0}$, porosity parameter $K_{P}$, Grashoff number for heat transfer $G_{r}$, Grashoff number for mass transfer $G_{c}$, Prandtl number $P_{r}$ and Schmidt number $S_{c}$. The expression for skin friction coefficient, Nusselt number and Sherwood number at the surface are also derived. The variations in the fluid velocity, temperature and concentration are shown graphically where as numerical values of skin-friction; Nusselt number and Sherwood number are presented in tabular form.
\end{abstract}

Key words-Chemical reaction, Heat source, MHD flow, Porous medium, Walters's $B^{\prime}$ model.

\section{Introduction}

The influence of magnetohydrodynamics heat and mass transfer processes over a moving surface is of interest in engineering and geophysical applications such as geothermal reservoirs, thermal insulation, enhanced oil recovery, packed-bed catalytic reaction and cooling of nuclear reactors. Many chemical engineering processes like metallurgical and polymer extrusion processes involve cooling of a molten liquid being stretched into a cooling system; the fluid mechanical properties of the penultimate product depend mainly on the cooling liquid used and the rate of stretching. Some polymer fluids like polyethylene oxide and polyisobutylene solution in cetane, having better electromagnetic properties are normally used as cooling liquid as their flow can be regulated by external magnetic fields in order to improve the quality of the final product.

Flows through porous media are frequently used in filtering of gasses, liquid and drying of bulk materials. In electrochemical engineering, porous electrodes and permeable, semi permeable diaphragms are used to obtain improved current efficiencies. In the field of agricultural engineering, porous media heat transfer plays an important role particularly in germinations of seeds. Above all man does a part of his breathing through his porous skin. More interestingly, application of magnetic field in the field of chemical engineering and agriculture attract many researchers to work on this new field of interest. Kronenberg rightly stated that "if the agricultural industry knew the benefit of magnetically treated water, the total agricultural output could be approximately increased by $25 \%$ \%. When fluid passes through a properly focus magnetic field, the magnetic field breaks up some of the complexes that are carried in the water, freeing the captive mineral particles. This process keeps minerals in the water rather than precipitated out. This is known as magnetic field conditioning. Further, magnetically treated fuel has a tendency to attract oxygen molecules when mixed with air in a combustion cylinder. This results a more efficient and complete combustion of the fuel generating more power from same amount of fuel. Research has shown that dairy cows will drink more magnetically treated water that untreated. This has increased milk production appreciably. With beef cattle, feed conversion ratio has increased by 5\% approximately enhancing meat production about the same. A chemical reaction involves in breaking of bonds in the reactive substances and formulation of bonds to form product species.

Several researchers have studied the two dimensional free convection, heat and mass transfer flow of an elastico- viscous fluid through porous medium. Sharma and Mathur [1] have studied the steady laminar free convection flow of an electrically conducting fluid along a porous hot vertical plate in the presence of heat source/ sink. Dash and Rath [2] have studied the problem of laminar flow and heat transfer of an electrically 
conducting fluid between parallel porous plates by applying explicit finite difference scheme. Walters [3] has studied the Non-Newtonian effects in some elastico-viscous liquids whose behavior at small rates of shear is characterized by a general linear equation of state. Unsteady two dimensional laminar flow of a viscous incompressible electrically conducting fluid in the vicinity of a semi-infinite vertical porous moving plate was discussed by Kim [4]. Flow and heat transfer of an electrically conducting visco-elastic fluid between two horizontal squeezing/ stretching plates has been studied by Rath et al. [5]. Unsteady MHD free convection and mass transfer flow past an infinite heated porous vertical plate with time dependent suction was discussed by Cookey and Sigalo [6].

Ece [7] has studied the free convection flow about a cone under mixed thermal boundary conditions and a magnetic field. Pravazinia and Nassehi [8] have studied the sheer thinning fluid flow through highly permeable porous media. Free convection heat and mass transfer from a horizontal cylinder of elliptic cross section in micro polar fluids has been studied by Yang [9]. Unsteady flow and heat transfer through an elasticoviscous liquid along an infinite hot vertical porous moving plate with variable free stream suction have been studied by Sharma and Pareek [10].MHD flow through a porous medium past a stretched vertical permeable surface in the presence of heat source/ sink and a chemical reaction have been studied by Dash et al. [11]. The effect of Hall current and chemical reaction on MHD flow along an exponentially porous flat plate with internal heat absorption/generation was studied by Rath et al. [12]. Free convective MHD flow through porous media of a rotating oldroyd fluid past an infinite vertical porous plate with heat and mass transfer have studied by Dash et al. [13]. Sharma and Sharma [14] have studied the unsteady two-dimensional flow and heat transfer through an elastico-viscous liquid along an infinite hot vertical porous surface bounded by porous medium.

The main objective of the present problem is to investigate the combined effect of transverse magnetic field, heat source and chemical reaction in the presence of oscillatory suction and free stream velocity on the flow, heat and mass transfer phenomena of an electrically conducting visco-elastic fluid.

\section{Formulation of the problem:}

An unsteady two dimensional MHD flow past a vertical infinite surface through a porous medium of a visco-elastic fluid (Walters fluid (model $B^{\prime}$ )) has been considered. The time dependent fluctuating suction velocity has been introduced with an oscillatory free stream velocity. A uniform transverse magnetic field $B_{0}$ is applied normal to the direction of the fluid flow. The $x^{*}$ - axis is taken along the surface in upward direction i.e. opposite to the direction of gravity and $y^{*}$-axis is taken normal to the surface.

The equations of continuity, motion, energy and diffusion for flow of a visco-elastic fluid through porous medium bounded by an infinite, hot vertical porous surface with oscillatory suction velocity are given by

$$
\begin{aligned}
& \frac{\partial v^{*}}{\partial y^{*}}=0, \\
& \rho\left(\frac{\partial u^{*}}{\partial t^{*}}+v^{*} \frac{\partial u^{*}}{\partial y^{*}}\right)=\rho \frac{\partial U^{*}}{\partial t^{*}}+\mu \frac{\partial^{2} u^{*}}{\partial y^{* 2}}-K_{0}^{*}\left(\frac{\partial^{3} u^{*}}{\partial t^{*} \partial y^{* 2}}+v^{*} \frac{\partial^{3} u^{*}}{\partial y^{* 3}}\right)+\frac{\mu}{K_{p}{ }^{*}}\left(U^{*}-u^{*}\right)+ \\
& \qquad B_{0}^{2}\left(U^{*}-u^{*}\right)+g \beta \rho\left(T^{*}-T_{\infty}\right)+g \beta^{*} \rho\left(C^{*}-C_{\infty}\right), \\
& \frac{\partial T^{*}}{\partial t^{*}}+v^{*} \frac{\partial T^{*}}{\partial y^{*}}=\frac{K}{\rho C_{P}} \frac{\partial^{2} T^{*}}{\partial y^{* 2}}+S^{*}\left(T^{*}-T_{\infty}\right), \\
& \frac{\partial C^{*}}{\partial t^{*}}+v^{*} \frac{\partial C^{*}}{\partial y^{*}}=D \frac{\partial^{2} C}{\partial y^{* 2}}-K_{C}^{*}\left(C^{*}-C_{\infty}\right) .
\end{aligned}
$$

Where $u^{*}, v^{*}$ are the velocity component along $x^{*}$ and $y^{*}$ direction respectively, $\rho$ the density of the fluid, $g$ the acceleration due to gravity, $\mu$ the dynamic viscosity, $K_{P}{ }^{*}$ the permeability parameter, $K_{O}{ }^{*}$ the non-Newtonian parameter, $K$ the thermal conductivity of the fluid, $C_{P}$ the specific heat of fluid at constant pressure, $K_{C}{ }^{*}$ the chemical reaction parameter, $S^{*}$ the heat source parameter, $T^{*}$ the temperature 
of the fluid, $\beta$ and $\beta^{*}$ are the volumetric coefficient of thermal and concentration expansion respectively, $\sigma$ the electrical conductivity, $C^{*}$ the concentration of the fluid, $D$ the mass diffusion co-efficient and $t^{*}$ the time.

The boundary conditions are:

$$
\begin{gathered}
y^{*}=0: u^{*}=0, T^{*}=T_{w}, C^{*}=C_{w} \\
y^{*} \rightarrow \infty: u^{*} \rightarrow U^{*}\left(t^{*}\right), T^{*} \rightarrow T_{\infty}, C^{*} \rightarrow C_{\infty}
\end{gathered}
$$

Where $T_{w}$ is the surface temperature, $U^{*}\left(t^{*}\right)$ the free stream velocity, $T_{\infty}$ is the free stream temperature, $C_{w}$ is the concentration at the wall and $C_{\infty}$ is the concentration of the fluid far away from the wall.

From equation of continuity (1) it is clear that the suction velocity normal to the plate $v^{*}$ is either a constant or a function of time. Hence, it is assumed that $v^{*}$ is in the form of

$$
v^{*}=-v_{0}\left(1+\varepsilon e^{i \omega^{*} t^{*}}\right)
$$

Where $\omega^{*}$ is the frequency of vibrations, $\varepsilon$ is the small parameter i.e., $(0<\varepsilon<1)$ and $v_{0}$ is a non zero positive suction velocity. The negative sign indicates that the suction is towards the plate.

To reduce the governing equations into non dimensional form, let us consider the following transformations.

$$
\begin{aligned}
& y=\frac{y^{*} v_{0}}{v}, t=\frac{t^{*} v_{0}{ }^{2}}{4 v}, u=\frac{u^{*}}{U}, \omega=\frac{4 v \omega^{*}}{v_{0}^{2}}, U(t)=\frac{U^{*}\left(t^{*}\right)}{U}, T=\frac{T^{*}-T_{\infty}}{T_{\omega}-T_{\infty}}, P_{r}=\frac{\mu C_{P}}{K}, K_{P}=\frac{K^{*} v_{0}^{2}}{v^{2}}, \\
& K_{0}=\frac{K^{*} v_{0}^{2}}{\rho v^{2}}, K_{C}=\frac{K_{C}{ }^{*} v}{v_{0}{ }^{2}}, S_{C}=\frac{v}{D}, M=\frac{B_{0}}{v_{0}} \sqrt{\frac{\sigma}{v \rho}}, C=\frac{C^{*}-C_{\infty}}{C_{w}-C_{\infty}}, S=\frac{S^{*} v}{v_{0}^{2}} \\
& G_{r}=\frac{v g \beta\left(T_{w}-T_{\infty}\right)}{U v_{0}^{2}}, G_{C}=\frac{v g \beta^{*}\left(C_{w}-C_{\infty}\right)}{U v_{0}^{2}}
\end{aligned}
$$

Where $G_{r}, G_{C}, P_{r}, S_{c}, K_{P}, K_{C}, M$ and $K_{0}$ are the Grashoff number for heat transfer, the Grashoff number for mass transfer, the Prandlt number, Schmidt number, the porosity parameter, the chemical reaction parameter, the magnetic parameter and the non-dimensional non-Newtonian parameter.

Using the transformation (7) equation (6) reduces to:

$$
v^{*}=-v_{0}\left(1+\varepsilon e^{i \omega t}\right)
$$

where $\omega$ is the frequency of the suction velocity.

In view of equation (7), equation (2) - (4) become

$$
\begin{aligned}
& \frac{1}{4} \frac{\partial u}{\partial t}-\left(1+\varepsilon e^{i \omega t}\right) \frac{\partial u}{\partial y}=\frac{\partial^{2} u}{\partial y^{2}}+m\left(1+\varepsilon e^{i \omega t}-u\right)-K_{0}\left\{\frac{1}{4} \frac{\partial^{3} u}{\partial t \partial y^{2}}-\left(1+\varepsilon e^{i \omega t}\right) \frac{\partial^{3} u}{\partial y^{3}}\right\} \\
& +\frac{1}{4} \varepsilon i \omega e^{i \omega t}+G_{r} T+G_{C} C \\
& \frac{P_{r}}{4} \frac{\partial T}{\partial t}-P_{r}\left(1+\varepsilon e^{i \omega t}\right) \frac{\partial T}{\partial y}=\frac{\partial^{2} T}{\partial y^{2}}+S T P_{r},
\end{aligned}
$$


$\frac{S_{C}}{4} \frac{\partial C}{\partial t}-S_{C}\left(1+\varepsilon e^{i \omega t}\right) \frac{\partial C}{\partial y}=\frac{\partial^{2} C}{\partial y^{2}}-K_{C} S_{C} C$,

where $m=M+\frac{1}{K_{P}}$ (say).

The corresponding boundary conditions in non- dimensional form are

$$
\begin{gathered}
y=0: u=0, T=1, C=1 \\
y \rightarrow \infty: u \rightarrow U(t), T \rightarrow 0, C \rightarrow 0
\end{gathered}
$$

In the neighborhood of the surface it is assumed that

$$
\begin{aligned}
& u(y, t)=u_{0}(y)+\varepsilon u_{1}(y) e^{i \omega t}, \\
& T(y, t)=T_{0}(y)+\varepsilon T_{1}(y) e^{i \omega t}, \\
& C(y, t)=C_{0}(y)+\varepsilon C_{1}(y) e^{i \omega t}
\end{aligned}
$$

and for the free stream $U(t)=1+\varepsilon e^{i \omega t}$.

Now, substituting (13) in the equations (9)-(11) and equating the coefficients of like power of $\varepsilon$ we get the following zeroth- order equations:

$$
\begin{aligned}
& K_{0} u_{0}^{\prime \prime \prime}+u_{0}^{\prime \prime}+u_{0}^{\prime}-m u_{0}=-G_{r} T_{0}-G_{C} C_{0}-m \\
& T_{0}^{\prime \prime}+P_{r} T_{0}^{\prime}+S P_{r} T_{0}=0 \\
& C_{0}^{\prime \prime}+S_{C} C_{0}^{\prime}-K_{C} S_{C} C_{0}=0
\end{aligned}
$$

First order equations are:

$$
\begin{aligned}
& K_{0} u_{1}^{\prime \prime \prime}+\left(1-\frac{K_{0} i \omega}{4}\right) u_{1}^{\prime \prime}+u_{1}^{\prime}-\left(m+\frac{i \omega}{4}\right) u_{1}=-G_{r} T_{1}-G_{C} C_{1}-\left(m+\frac{i \omega}{4}\right)-K_{0} u_{0}^{\prime \prime \prime}-u_{0}^{\prime} \\
& T_{1}^{\prime \prime}+P_{r} T_{1}^{\prime}+\left(P_{r} S-\frac{P_{r} i \omega}{4}\right) T_{1}=-P_{r} T_{0}^{\prime} \\
& C_{1}^{\prime \prime}+S_{C} C_{1}^{\prime}-\left(\frac{S_{C} i \omega}{4}+K_{C} S_{C}\right) C_{1}=-S_{C} C_{0}^{\prime}
\end{aligned}
$$

where prime denotes the differentiation with respect to $y$.

The corresponding boundary conditions are changed to

$$
\begin{aligned}
& y=0: u_{0}=0, u_{1}=0, T_{0}=1, T_{1}=0, C_{0}=1, C_{1}=0 \\
& y \rightarrow \infty: u_{0} \rightarrow 1, u_{1} \rightarrow 1, T_{0} \rightarrow 0, T_{1} \rightarrow 0, C_{0} \rightarrow 0, C_{1} \rightarrow 0
\end{aligned}
$$

Solving equations (15), (16), (18) and (19) using the boundary conditions (20), we get

$$
\begin{aligned}
& T_{0}=e^{m_{2} y}, \\
& C_{0}=e^{m_{6} y}, \\
& T_{1}=I_{1}\left(e^{m_{4} y}-e^{m_{2} y}\right),
\end{aligned}
$$




$$
C_{1}=I_{2}\left(e^{m_{8} y}-e^{m_{6} y}\right)
$$

where $I_{1}=\frac{P_{r} m_{2}}{m_{2}^{2}+P_{r} m_{2}+S P_{r}-\frac{i \omega}{4} P_{r}}$ and $I_{2}=\frac{4 i m_{6}}{\omega}$

But equation (14) and (17) are of third order equations when $K_{0} \neq 0$ and these equations reduce to second order equations when $K_{0}=0$ (for Newtonian fluid). Hence the presence of non-Newtonian parameter increases the order of the differential equation. As the non-Newtonian parameter $\left(K_{0}\right)$ is very small for incompressible fluid (Walters), therefore $u_{0}$ and $u_{1}$ can be expanded in powers of $K_{0}$ which are given by

$u_{0}(y)=u_{00}(y)+K_{0} u_{01}(y)+o\left(K_{0}^{2}\right)$
$u_{1}(y)=u_{10}(y)+K_{0} u_{11}(y)+o\left(K_{0}^{2}\right)$

Introducing (25) into the equations (14) and (17) and equating like powers of $K_{0}$ in both sides we get

$$
\begin{aligned}
& u_{00}^{\prime \prime}+u_{00}^{\prime}-m u_{00}=-G_{r} T_{0}-G_{C} C_{0}-m \\
& u_{10}^{\prime \prime}+u_{10}^{\prime}-\left(m+\frac{i \omega}{4}\right) u_{10}=-G_{r} T_{1}-G_{C} C_{1}-\left(m+\frac{i \omega}{4}\right)-u_{00}^{\prime} \\
& u_{01}^{\prime \prime}+u_{01}^{\prime}-m u_{01}=-u_{00}^{\prime \prime \prime} \\
& u_{11}^{\prime \prime}+u_{11}^{\prime}-\left(m+\frac{i \omega}{4}\right) u_{11}=-u_{10}^{\prime \prime \prime}+u_{10}^{\prime \prime} \frac{i \omega}{4}-u_{00}^{\prime \prime \prime}-u_{01}^{\prime}
\end{aligned}
$$

The boundary conditions are

$$
\begin{aligned}
& y=0: u_{00}=0, u_{01}=0, u_{10}=0, u_{11}=0 \\
& y \rightarrow \infty: u_{00} \rightarrow 1, u_{01} \rightarrow 0, u_{10} \rightarrow 1, u_{11} \rightarrow 0
\end{aligned}
$$

Solving equations (26)-(29) using the boundary condition (30), we get

$$
\begin{aligned}
& u_{00}=I_{3} e^{m_{10} y}-I_{4} e^{m_{2} y}-I_{5} e^{m_{6} y}+1 \\
& u_{01}=I_{9} e^{m_{10} y}+I_{6} y e^{m_{10} y}+I_{7} e^{m_{2} y}+I_{8} e^{m_{6} y} \\
& u_{10}=I_{16} e^{m_{12} y}+I_{17} e^{m_{2} y}-I_{18} e^{m_{4} y}+I_{19} e^{m_{6} y}-I_{20} e^{m_{8} y}-I_{21} e^{m_{10} y}+1 \\
& u_{11}=I_{35} e^{m_{12} y}+I_{28} e^{m_{2} y}+I_{29} e^{m_{4} y}+I_{30} e^{m_{6} y}+I_{31} e^{m_{8} y}+I_{32} e^{m_{10} y}+I_{33} e^{m_{12} y} y-I_{34} e^{m_{10} y} y
\end{aligned}
$$

Hence the velocity $u(y, t)$ is given by

$$
\begin{aligned}
u(y, t)= & I_{3} e^{m_{10} y}-I_{4} e^{m_{2} y}-I_{5} e^{m_{6} y}+1+K_{0}\left\{I_{9} e^{m_{10} y}+I_{6} y e^{m_{10} y}+I_{7} e^{m_{2} y}+I_{8} e^{m_{6} y}\right\} \\
& +\varepsilon\left[I_{16} e^{m_{12} y}+I_{17} e^{m_{2} y}-I_{18} e^{m_{4} y}+I_{19} e^{m_{6} y}-I_{20} e^{m_{8} y}-I_{21} e^{m_{10} y}+1\right. \\
& +K_{0}\left\{I_{35} e^{m_{12} y}+I_{28} e^{m_{2} y}+I_{29} e^{m_{4} y}+I_{30} e^{m_{6} y}+I_{31} e^{m_{8} y}+I_{32} e^{m_{10} y}\right. \\
& \left.\left.+I_{33} e^{m_{12} y} y-I_{34} e^{m_{10} y} y\right\}\right] e^{i \omega t}
\end{aligned}
$$

Where $I_{3}-I_{35}, m_{1}, \ldots \ldots \ldots . . . ., m_{12}$ are given in the appendix.

Skin friction $\left(C_{f}\right)$ 


$$
C_{f}=\frac{\tau_{\omega}^{*}}{\rho U \nu}=\left[\frac{\partial u}{\partial y}-K_{0}\left(\frac{1}{4} \frac{\partial^{2} u}{\partial t \partial y}-\left(1+\varepsilon e^{i \omega t}\right) \frac{\partial^{2} u}{\partial y^{2}}\right)\right]_{y=0}
$$

Nusselt Number $\left(N_{u}\right)$

$$
\left.N_{u}=\frac{q_{1} v}{v_{0} K\left(T_{\omega}-T \infty\right)}=-\frac{\partial T}{\partial y}\right]_{y=0}=-\left[m_{2}+\varepsilon e^{i \omega t} I_{1}\left(m_{4}-m_{2}\right)\right]
$$

Sherwood Number $\left(S_{h}\right)$

$$
\left.S_{h}=\frac{q_{2} \nu}{v_{0} D\left(C_{\omega}-C_{\infty}\right)}=-\frac{\partial C}{\partial y}\right]_{y=0}=-\left[m_{6}+\varepsilon e^{i \omega t} I_{2}\left(m_{8}-m_{6}\right)\right]
$$

Where $\tau_{\omega}^{*}$ is the dimension shear Stress component of the elastico-viscous fluid and the symbols $q_{1}$ and $q_{2}$ represent heat and mass flux and these are given by

$q_{1}=-K\left(\frac{\partial T^{*}}{\partial y^{*}}\right)_{y^{*}=0} \quad$ and $\quad q_{2}=-D\left(\frac{\partial C^{*}}{\partial y^{*}}\right)_{y^{*}=0}$

\section{Results and Discussion:}

The following discussions bring out the effects of porosity parameter $\left(K_{P}\right)$, Source parameter $(S)$, Grashoff number for heat transfer $\left(G_{r}\right)$, Grashoff number for mass transfer $\left(G_{c}\right)$, prandatl number $\left(P_{r}\right)$, Schmidt number $\left(S_{c}\right)$, chemical reaction parameter $\left(K_{c}\right)$, magnetic parameter $(M)$, phase angle $(\omega t)$ and elastic parameter $\left(K_{0}\right)$.

Fig.1 shows the velocity distribution for various values of $G_{r}, G_{c}, K_{0}, K_{p}, P_{r}, S_{c}, \omega, \omega t$, and $M$. The common characteristics of all the curves are to increase the velocity with the layers near the vertical surfaces. From the curves (III, IV, V, VI) it is observed that an increase in $G_{r}$ increases the velocity near the plate. It is due to free convection current which accelerates the velocity near the plate. Further higher Prandtl number fluid contributes very insignificantly in decreasing the velocity (curves III, VII). It is further to note that the presence of transverse magnetic field reduces the velocity due to generated Lorentz's force opposing the motion (curves III, XIII). It is also observed that fluid velocity increases due to increase in the elastic parameter $\left(K_{0}\right)$, Grashoff number for mass transfer $\left(G_{c}\right)$, chemical reaction parameter $\left(K_{c}\right)$, and Source parameter $(S)$ but reverse effect is observed in case of porosity parameter $\left(K_{P}\right)$, phase angle $(\omega t)$, and $\operatorname{Schmidt}$ number $\left(S_{c}\right)$.

$\begin{array}{lccccccccccc}\text { Curve } & G_{r} & P_{r} & K_{p} & \omega & \omega t & K_{0} & G_{c} & M & K_{c} & S_{c} & S \\ -1 & 5 & 7 & 0.4 & 5 & \pi / 4 & 0 . & 0 & 0 & 0 & 0.22 & 1 \\ \text { II } & 5 & 7 & 0.4 & 5 & \pi / 4 & 0.2 & 0 & 0 & 0 & 0.22 & 1 \\ \text { III } & 5 & 7 & 0.4 & 5 & \pi / 4 & 0.2 & 2 & 1 & 1 & 0.22 & 1 \\ \text { IV } & 10 & 7 & 0.4 & 5 & \pi / 4 & 0.2 & 2 & 1 & 1 & 0.22 & 1 \\ \text { V } & -5 & 7 & 0.4 & 5 & \pi / 4 & 0.2 & 2 & 1 & 1 & 0.22 & 1 \\ \text { VI } & -10 & 7 & 0.4 & 5 & \pi / 4 & 0.2 & 2 & 1 & 1 & 0.22 & 1 \\ \text { VII } & 5 & 11.4 & 0.4 & 5 & \pi / 4 & 0.2 & 2 & 1 & 1 & 0.22 & 1 \\ \text { VIII } & 5 & 7 & 100 & 5 & \pi / 4 & 0.2 & 2 & 1 & 1 & 0.22 & 1 \\ \text { IX } & 5 & 7 & 0.4 & 15 & \pi / 4 & 0.2 & 2 & 1 & 1 & 0.22 & 1 \\ \text { X } & 5 & 7 & 0.4 & 5 & \pi / 3 & 0.2 & 2 & 1 & 1 & 0.22 & 1 \\ \text { XI } & 5 & 7 & 0.4 & 5 & \pi / 4 & 0.0 & 2 & 1 & 1 & 0.22 & 1 \\ \text { XII } & 5 & 7 & 0.4 & 5 & \pi / 4 & 0.2 & 3 & 1 & 1 & 0.22 & 1 \\ \text { XIII } & 5 & 7 & 0.4 & 5 & \pi / 4 & 0.2 & 2 & 5 & 1 & 0.22 & 1 \\ \text { XIV } & 5 & 7 & 0.4 & 5 & \pi / 4 & 0.2 & 2 & 1 & -.04 & 0.22 & 1 \\ \text { XV } & 5 & 7 & 0.4 & 5 & \pi / 4 & 0.2 & 2 & 1 & 1 & 0.78 & 1 \\ \text { XVI } & 5 & 7 & 0.4 & 5 & \pi / 4 & 0.2 & 2 & 1 & 1 & 0.22 & 0 \\ \text { XVII } & 5 & 7 & 0.4 & 5 & \pi / 4 & 0.2 & 2 & 1 & 1 & 0.22 & 1.5\end{array}$




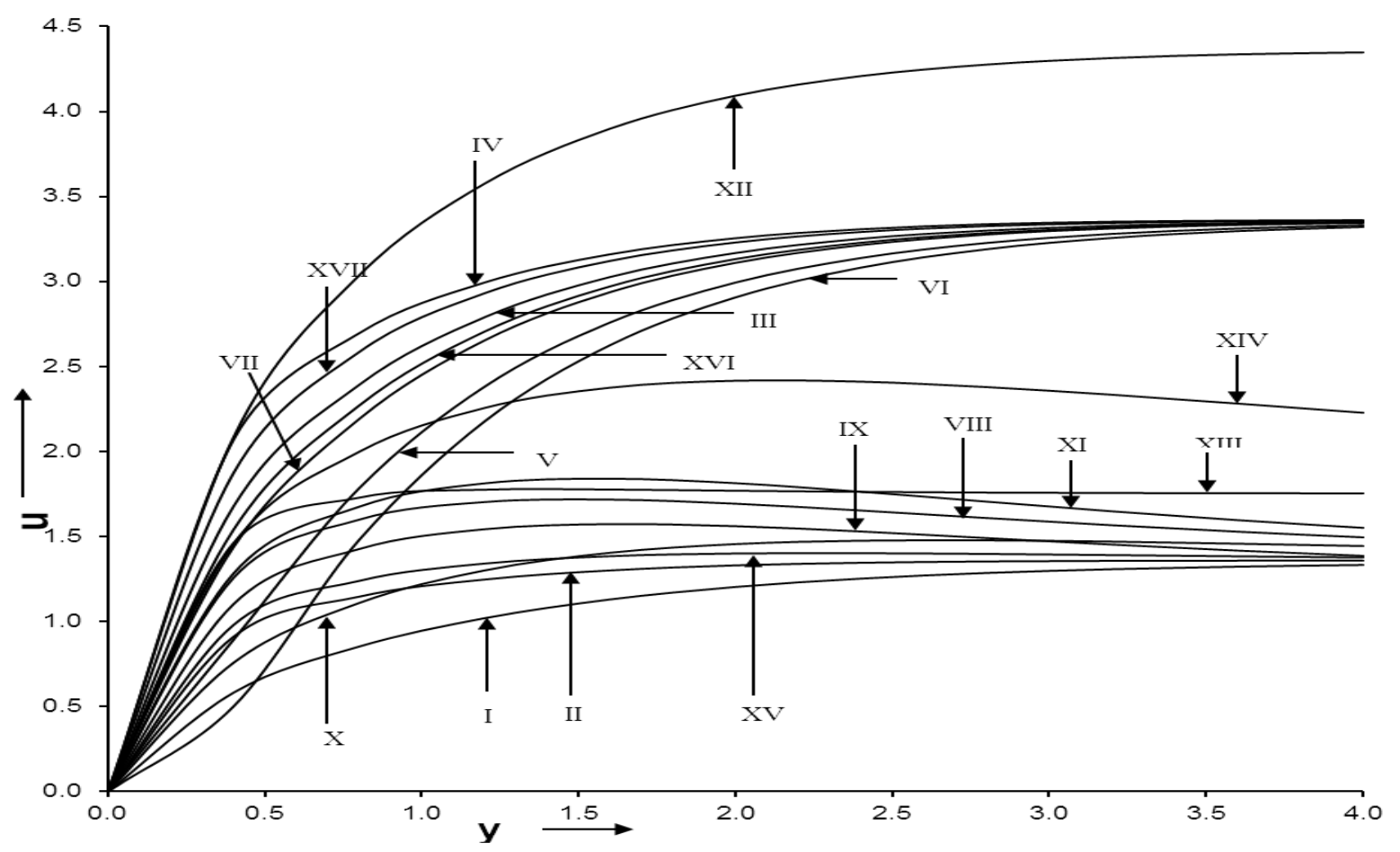

Fig- 1 Effect of different parameters on Velocity profile when $\varepsilon=0.5$

Fig. 2 depicts the temperature distribution in the flow domain. An increase in heat source parameter (S) contribute to increase the thickness of the thermal boundary layer (curve I, V, VI) but with an increase in the value of prandtl number $\left(P_{r}\right)$, frequency parameter $(\omega)$ and phase angle $(\omega t)$ the temperature decreases (curves I, II, III and IV). This may be attributed to the fact that smaller values of $P_{r}$ lead to increase the thermal conductivity of the fluid and there by more amount of heat is diffused which causes the decrease in temperature.

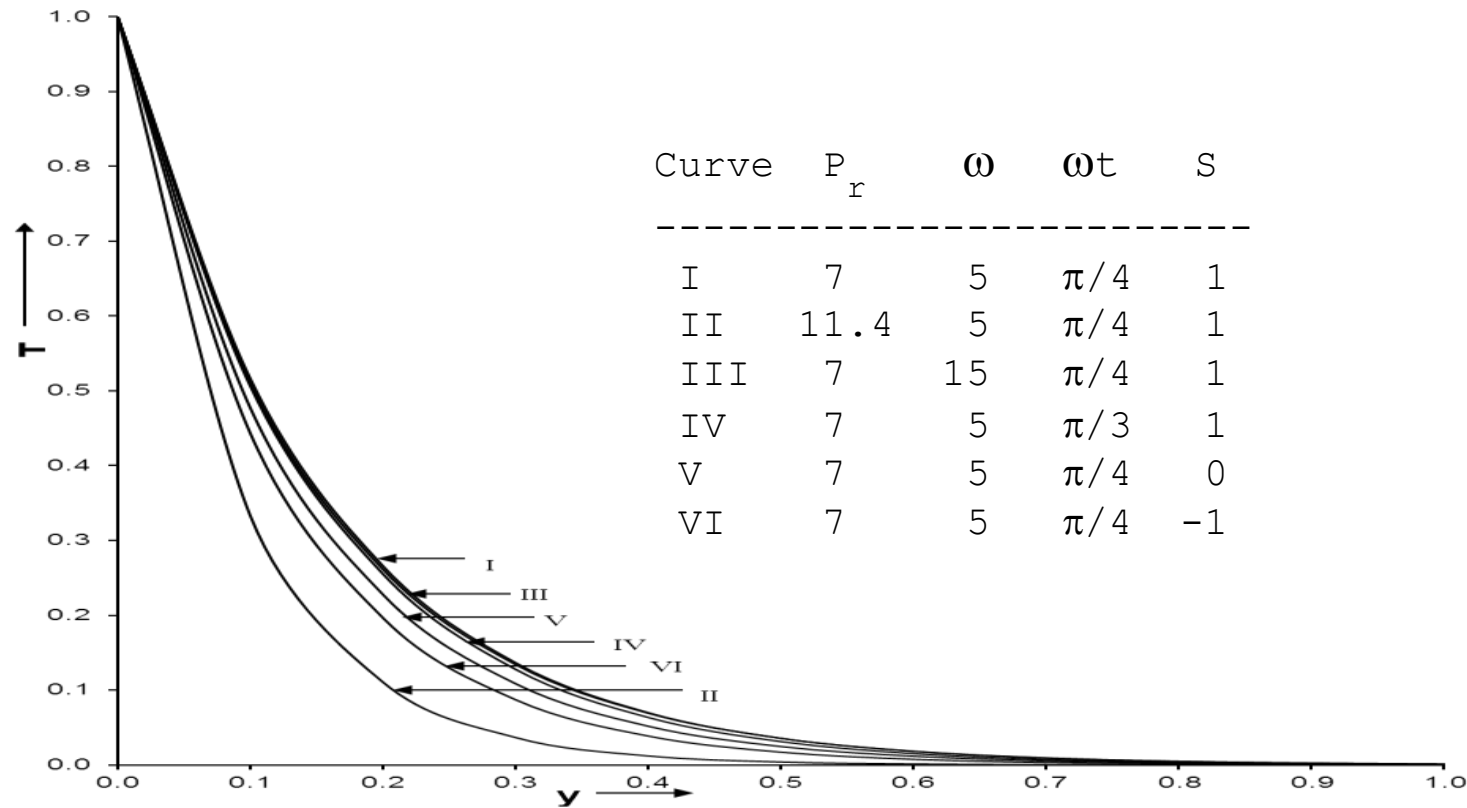

Fig- 2 Temperature profile when $\square=0.5$

Fig.3 exhibits the concentration distribution in the flow field. An increase in Schmidt number $\left(S_{C}\right)$ and chemical reaction parameter $\left(K_{C}\right)$ leads to decrease the concentration. Also an increase in frequency of oscillation $(\omega)$ leads to increase the concentration (curves I,VI).It is also interesting to note that phase angle has no impact on the concentration profile as all the curves coincide with each other (curves I, VII). 


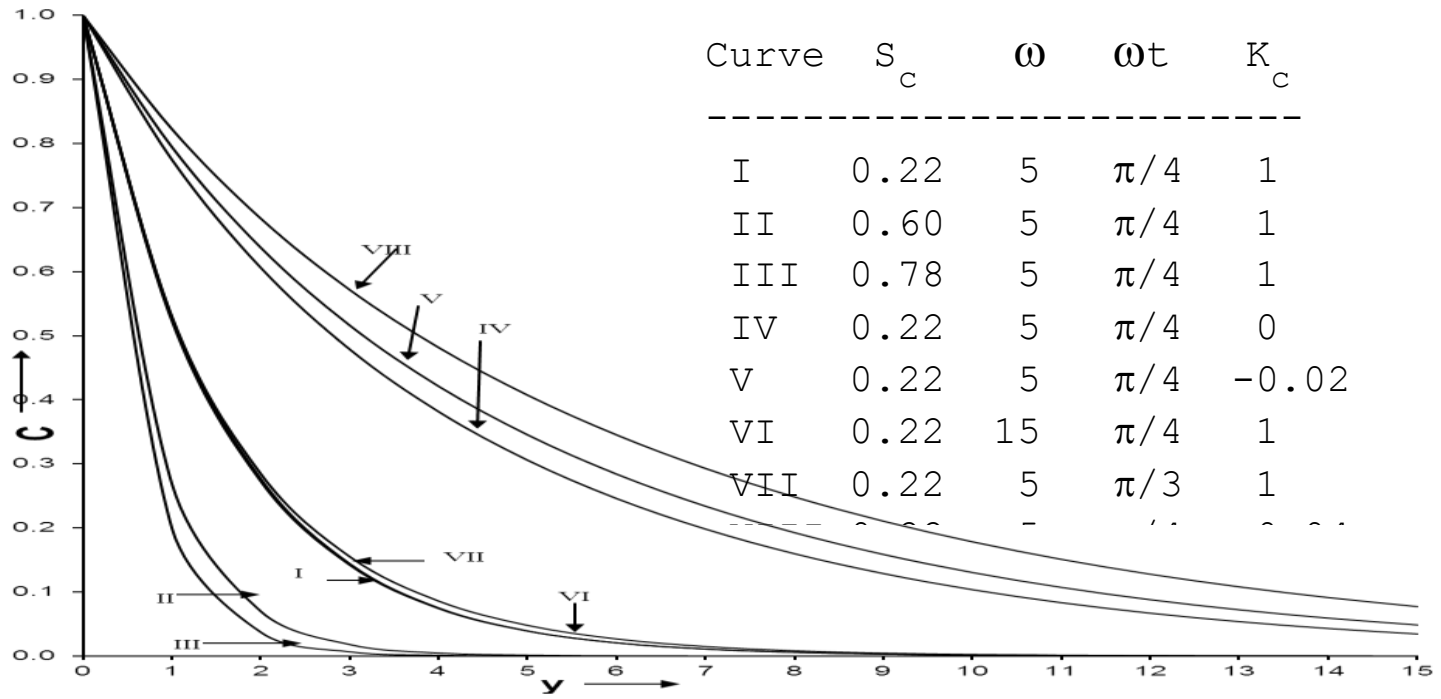

Fig- 3 Concentration profile when $\square=0.5$

Table -1 Skin friction $\left(C_{f}\right)$

\begin{tabular}{|c|c|c|c|c|c|c|c|c|c|c|c|}
\hline $\boldsymbol{G}_{\boldsymbol{r}}$ & $\boldsymbol{P}_{\boldsymbol{r}}$ & $\boldsymbol{K}_{\boldsymbol{p}}$ & $\boldsymbol{\omega}$ & $\boldsymbol{\omega} \boldsymbol{t}$ & $\boldsymbol{K}_{\boldsymbol{o}}$ & $\boldsymbol{G}_{\boldsymbol{c}}$ & $\boldsymbol{M}$ & $\boldsymbol{K}_{\boldsymbol{c}}$ & $\boldsymbol{S}_{\boldsymbol{c}}$ & $\boldsymbol{S}$ & $\boldsymbol{C}_{\boldsymbol{f}}$ \\
\hline 5 & 7 & 0.4 & 5 & $\pi / 4$ & 0 & 0 & 0 & 0 & 0.22 & 1 & 13.40476 \\
\hline 5 & 7 & 0.4 & 5 & $\pi / 4$ & 0.2 & 0 & 0 & 0 & 0.22 & 1 & 35.80533 \\
\hline 5 & 7 & 0.4 & 5 & $\pi / 4$ & 0.2 & 2 & 1 & 1 & 0.22 & 1 & 40.87522 \\
\hline 10 & 7 & 0.4 & 5 & $\pi / 4$ & 0.2 & 2 & 1 & 1 & 0.22 & 1 & 74.87893 \\
\hline-5 & 7 & 0.4 & 5 & $\pi / 4$ & 0.2 & 2 & 1 & 1 & 0.22 & 1 & 27.13221 \\
\hline-10 & 7 & 0.4 & 5 & $\pi / 4$ & 0.2 & 2 & 1 & 1 & 0.22 & 1 & 61.13592 \\
\hline 5 & 11.4 & 0.4 & 5 & $\pi / 4$ & 0.2 & 2 & 1 & 1 & 0.22 & 1 & 45.64340 \\
\hline 5 & 7 & 100 & 5 & $\pi / 4$ & 0.2 & 2 & 1 & 1 & 0.22 & 1 & 40.87522 \\
\hline 5 & 7 & 0.4 & 15 & $\pi / 4$ & 0.2 & 2 & 1 & 1 & 0.22 & 1 & 43.06077 \\
\hline 5 & 7 & 0.4 & 5 & $\pi / 3$ & 0.2 & 2 & 1 & 1 & 0.22 & 1 & 37.98120 \\
\hline 5 & 7 & 0.4 & 5 & $\pi / 4$ & 0 & 2 & 1 & 1 & 0.22 & 1 & 15.75162 \\
\hline 5 & 7 & 0.4 & 5 & $\pi / 4$ & 0.2 & 3 & 1 & 1 & 0.22 & 1 & 42.12474 \\
\hline 5 & 7 & 0.4 & 5 & $\pi / 4$ & 0.2 & -2 & 1 & 1 & 0.22 & 1 & 35.87712 \\
\hline 5 & 7 & 0.4 & 5 & $\pi / 4$ & 0.2 & 2 & 5 & 1 & 0.22 & 1 & 61.66407 \\
\hline 5 & 7 & 0.4 & 5 & $\pi / 4$ & 0.2 & 2 & 1 & 0 & 0.22 & 1 & 42.62096 \\
\hline 5 & 7 & 0.4 & 5 & $\pi / 4$ & 0.2 & 2 & 1 & -0.04 & 0.22 & 1 & 42.93118 \\
\hline 5 & 7 & 0.4 & 5 & $\pi / 4$ & 0.2 & 2 & 1 & 1 & 0.78 & 1 & 39.99505 \\
\hline 5 & 7 & 0.4 & 5 & $\pi / 4$ & 0.2 & 2 & 1 & 1 & 0.22 & 0 & 40.09924 \\
\hline 5 & 7 & 0.4 & 5 & $\pi / 4$ & 0.2 & 2 & 1 & 1 & 0.22 & 1.5 & 41.79616 \\
\hline
\end{tabular}


Table 1 shows the numerical values of skin friction which is very useful from the application point of view. From table 1 it is observed that the coefficient of skin friction increases due to increase in $G_{r}, P_{r}, G_{C}, \omega$, $K_{C}, M$ and $S$ whereas it decreases due to the presence of $S_{C}, K_{C}$ and $\omega t$.

From table 2 it is observed that the rate of heat transfer $\left(N_{u}\right)$ at the surface increases in case of higher prandatl number $\left(P_{r}\right)$, frequency of oscillation $(\omega)$ and phase angle $(\omega t)$ but it decreases due to the presence of heat source parameter $(S)$.

From table 3 it can be seen that the rate of mass transfer $\left(S_{h}\right)$ at the surface increases with an increase in $\mathrm{K}_{\mathrm{C}}>0$ and $\mathrm{S}_{\mathrm{C}}$ but frequency $(\omega)$ and phase angle $(\omega t)$ has no significant effect. In case of generative reaction $\left(K_{C}<0\right)$ it increases with an increase in the value of $K_{C}$.

Table - 2 Nusselt number $\left(\mathbf{N}_{\mathrm{u}}\right)$

\begin{tabular}{|c|c|c|c|c|}
\hline $\boldsymbol{P}_{\boldsymbol{r}}$ & $\boldsymbol{\omega}$ & $\boldsymbol{\omega t}$ & $\boldsymbol{S}$ & $\boldsymbol{N}_{\boldsymbol{u}}$ \\
\hline 7 & 5 & $\pi / 4$ & 1 & 6.59168 \\
\hline 11.4 & 5 & $\pi / 4$ & 1 & 10.96700 \\
\hline 7 & 15 & $\pi / 4$ & 1 & 6.72523 \\
\hline 7 & 5 & $\pi / 3$ & 1 & 6.77149 \\
\hline 7 & 5 & $\pi / 4$ & 0 & 7.38594 \\
\hline 7 & 5 & $\pi / 4$ & -1 & 8.12559 \\
\hline
\end{tabular}

Table - 3 Sherwood number $\left(S_{h}\right)$

\begin{tabular}{|c|c|c|c|c|}
\hline $\boldsymbol{S}_{\boldsymbol{c}}$ & $\boldsymbol{\omega}$ & $\boldsymbol{\omega t}$ & $\boldsymbol{K}_{\boldsymbol{c}}$ & $\boldsymbol{S}_{\boldsymbol{h}}$ \\
\hline 0.22 & 5 & $\pi / 4$ & 1 & 0.64448 \\
\hline 0.60 & 5 & $\pi / 4$ & 1 & 1.29035 \\
\hline 0.78 & 5 & $\pi / 4$ & 1 & 1.56971 \\
\hline 0.22 & 5 & $\pi / 4$ & 0 & 0.25931 \\
\hline 0.22 & 5 & $\pi / 4$ & -0.02 & 0.23432 \\
\hline 0.22 & 15 & $\pi / 4$ & 1 & 0.63724 \\
\hline 0.22 & 5 & $\pi / 3$ & 1 & 0.63443 \\
\hline 0.22 & 5 & $\pi / 4$ & -0.04 & 0.19985 \\
\hline
\end{tabular}

\section{Conclusion}

1. Effect of elasticity on velocity depends on heating or cooling of the surface, being opposite to each other, whereas effect of other parameters are almost independent of heating or cooling of the plate.

2. A flow characteristic in the present study is more dependent on mass buoyancy effect $\left(\mathrm{G}_{\mathrm{C}}\right)$ rather than thermal buoyancy effect $\left(\mathrm{G}_{\mathrm{r}}\right)$.

3. Thinning of thermal boundary layer occurs for higher prandtl number fluid.

4. Higher rate of mass transfer is experienced in case of heavier species and in the presence of destructive reaction. 


\section{Reference:}

[1] P.R.Sharma and P. Mathur, Study on laminar free convection flow of an electrically conducting fluid along a porous hot vertical plate in the presence of heat source /sink, Indian journal of pure applied mathematics), 26, 1995,11251134.

[2] G.C. Dash and P.K. Rath, Explicit finite difference scheme for flow and heat transfer of an electrically conducting fluid between parallel porous plates, Proc.National Academy of Science India, 67(II), 1997,185-192.

[3] K. Walters, Non-Newtonian effects in some elastic-viscous liquids whose behavior at small rates of shear is characterized by a general linear equation of state, Quart. J. Mech. Appl. Math, 15, 1962, 63-76.

[4] Y. J.Kim, Unsteady convective heat transfer past a semi -infinite vertical porous moving plate with variable suction, International journal of engineering Science, 38, 2000, 833-845.

[5] P.K.Rath, G.C.Dash and P.K. Rath, Flow and heat transfer of an electrically conducting visco-elastic fluid between two horizontal squeezing / stretching plates, Association for the Advancement of Modeling and Simulation Techniques in Enterprises, Modeling Measurement and Control, B 70( 6) , 2001,45 -63 .

[6] C.Israel-cookey and F B Sigalo, Unsteady MHD free convection and mass transfer flow past an infinite heated porous vertical plate with time dependent suction, Association for the Advancement of Modeling and Simulation Techniques in Enterprises, Modeling Measurement and Control, B72, 2003,25-38.

[7] M.C.Ece, Free convection flow about a cone under mixed thermal boundary conditions and a magnetic field, Applied mathematical modeling, 29, 2005,1121- 1134.

[8] M.Prrvazinia and V. Nussehi, Study of shear thinning fluid flow through highly permeable porous media, International communication in Heat and mass transfer, 33, 2006, $401-410$.

[9] C.C.Yang, Free convection heat and mass transfer from a horizontal cylinder of an elliptic cross section in micro polar fluids, International communication in Heat and mass transfer, 33, 2006, 311-318.

[10] P.R.Sharma, and D. Pareek, Unsteady flow and Heat transfer through an elastico - viscous liquid along an infinite hot vertical porous moving plate with variable free stream suction, Bulletin of Calcutta Mathematical Society, 98, 2006, 97-108.

[11] S. Dash, G.C. Dash and D.P. Mishra, MHD flow through a porous medium past a stretched vertical preamble surface in the presence of heat source /sink and a chemical reaction, Proc.National Academy of Science India, 78(A), (I ), 2008, 49-55.

[12] P.K. Rath, G.C. Dash and A.K. Patra, Effect of Hall current and chemical reaction on MHD flow along an exponentially accelerated porous flat plate with internal heat heat absorption /generation, Proc.National Academy of Science India, 80(A), 2010, 295-308.

[13] G.C. Dash, P.K. Rath and M. Kar, Free convective MHD flow through porous media of a rotating oldroyd fluid past an infinite vertical porous plate with heat and mass transfer, Proc.National Academy of Science India, 81 (A), pp. 2011.

[14] P.R.Sharma and S.Sharma, Unsteady two dimensional flow and heat transfer through an elastico-viscous liquid along an infinite hot vertical porous surface bounded by porous medium, Bulletin of Calcutta Mathematical Society, 97, $2005,477-488$.

\section{Appendix}

$$
\begin{aligned}
& m_{1}=\frac{-P_{r}+\sqrt{P_{r}^{2}-4 S P_{r}}}{2}, m_{2}=\frac{-P_{r}-\sqrt{P_{r}^{2}-4 S P_{r}}}{2}, m_{3}=\frac{-P_{r}+\sqrt{P_{r}^{2}-4\left(P_{r} S-\frac{P_{r} i \omega}{4}\right)}}{2} \\
& m_{4}=\frac{-P_{r}-\sqrt{P_{r}^{2}-4\left(P_{r} S-\frac{P_{r} i \omega}{4}\right)}}{2}, m_{5}=\frac{-S_{C}+\sqrt{S_{C}^{2}+4 S_{C} K_{C}}}{2} \\
& m_{6}=\frac{-S_{C}-, \sqrt{S_{C}^{2}+4 S_{C} K_{C}}}{2}, m_{7}=\frac{-S_{C}+\sqrt{S_{C}^{2}+4\left(\frac{S_{C} i \omega}{4}+K_{C} S_{C}\right)}}{2} \\
& m_{8}=\frac{-S_{C}-\sqrt{S_{C}^{2}+4\left(\frac{S_{C} i \omega}{4}+K_{C} S_{C}\right)}}{2}, m_{9}=\frac{-1+\sqrt{1+4 m}}{2}, m_{10}=\frac{-1-\sqrt{1+4 m}}{2} \\
& m_{11}=\frac{-1+\sqrt{1+4 I_{15}}}{2}, \quad m_{12}=\frac{-1-\sqrt{1+4 I_{15}}}{2} \\
& I_{3}=\frac{G_{r}}{m_{2}^{2}+m_{2}-m}+\frac{G_{C}}{m_{6}^{2}+m_{6}-m}-1, I_{4}=\frac{G_{r}}{m_{2}^{2}+m_{2}-m}, I_{5}=\frac{G_{C}}{m_{6}^{2}+m_{6}-m} \\
& I_{6}=\frac{-I_{3}\left(m_{10}\right)^{3}}{2 m_{10}+1}, I_{7}=\frac{I_{4}\left(m_{2}\right)^{3}}{m_{2}^{2}+m_{2}-m}, I_{8}=\frac{I_{5}\left(m_{6}\right)^{3}}{m_{6}^{2}+m_{6}-m}, I_{9}=-\left(I_{7}+I_{8}\right),
\end{aligned}
$$




$$
\begin{aligned}
& I_{10}=G_{r} I_{1}+I_{4} m_{2}, I_{11}=G_{r} I_{1}, I_{12}=G_{C} I_{2}+I_{5} m_{6}, I_{13}=G_{C} I_{2}, I_{14}=I_{3} m_{10}, \\
& I_{15}=m+\frac{i \omega}{4}, \\
& I_{16}=\frac{-I_{10}}{m_{2}^{2}+m_{2}-I_{15}}+\frac{I_{11}}{m_{4}^{2}+m_{4}-I_{15}}-\frac{-I_{12}}{m_{6}^{2}+m_{6}-I_{15}}+\frac{I_{13}}{m_{8}^{2}+m_{8}-I_{15}}+\frac{I_{14}}{m_{10}^{2}+m_{10}-I_{15}}-1 \\
& I_{17}=\frac{I_{10}}{m_{2}^{2}+m_{2}-I_{15}}, I_{18}=\frac{I_{11}}{m_{4}^{2}+m_{4}-I_{15}}, I_{19}=\frac{I_{12}}{m_{6}^{2}+m_{6}-I_{15}} \\
& I_{20}=\frac{I_{13}}{m_{8}^{2}+m_{8}-I_{15}}, I_{21}=\frac{I_{14}}{m_{10}^{2}+m_{10}-I_{15}} \\
& I_{22}=I_{4} m_{2}^{3}-I_{7} m_{2}-I_{17} m_{2}^{3}+\frac{i \omega}{4} I_{17} m_{2}^{2}, I_{23}=I_{18} m_{4}^{3}-I_{18} m_{4}^{2} \frac{i \omega}{4}, \\
& I_{24}=I_{5} m_{6}^{3}-I_{8} m_{6}-I_{19} m_{6}^{3}+\frac{i \omega}{4} I_{19} m_{6}^{2}, I_{25}=I_{20} m_{8}^{3}-I_{20} m_{8}^{2} \frac{i \omega}{4}, \\
& I_{26}=I_{21} m_{10}^{3}-\frac{i \omega}{4} I_{21} m_{10}^{2}-I_{3} m_{10}^{3}-I_{9} m_{10}-I_{6}, I_{27}=-I_{16} m_{12}^{3}+I_{16} m_{12}^{2} \frac{i \omega}{4} \\
& I_{28}=\frac{I_{22}}{m_{2}^{2}+m_{2}-I_{15}}, I_{29}=\frac{I_{23}}{m_{4}^{2}+m_{4}-I_{15}}, I_{30}=\frac{I_{24}}{m_{6}^{2}+m_{6}-I_{15}}, I_{31}=\frac{I_{25}}{m_{8}^{2}+m_{8}-I_{15}}, \\
& I_{32}=\frac{I_{26}}{m_{10}^{2}+m_{10}-I_{15}}+\frac{I_{6} m_{10}\left(2 m_{10}+1\right)}{\left(m_{10}^{2}+m_{10}-I_{15}\right)^{2}}, I_{33}=\frac{I_{27}}{2 m_{12}+1}, I_{34}=\frac{I_{6} m_{10}}{m_{10}^{2}+m_{10}-I_{15}}, \\
& I_{35}=-\left(I_{28}+I_{29}+I_{30}+I_{31}+I_{32}\right)
\end{aligned}
$$

